\title{
Engine performance analysis and optimization of a dual-mode scramjet with varied inlet conditions
}

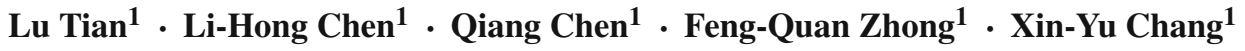

Received: 25 June 2014 / Revised: 28 January 2015 / Accepted: 7 April 2015 / Published online: 16 September 2015

(C) The Chinese Society of Theoretical and Applied Mechanics; Institute of Mechanics, Chinese Academy of Sciences and Springer-Verlag Berlin Heidelberg 2015

\begin{abstract}
A dual-mode scramjet can operate in a wide range of flight conditions. Higher thrust can be generated by adopting suitable combustion modes. Based on the net thrust, an analysis and preliminary optimal design of a kerosene-fueled parameterized dual-mode scramjet at a crucial flight Mach number of 6 were investigated by using a modified quasi-one-dimensional method and simulated annealing strategy. Engine structure and heat release distributions, affecting the engine thrust, were chosen as analytical parameters for varied inlet conditions (isolator entrance Mach number: 1.5-3.5). Results show that different optimal heat release distributions and structural conditions can be obtained at five different inlet conditions. The highest net thrust of the parameterized dual-mode engine can be achieved by a subsonic combustion mode at an isolator entrance Mach number of 2.5. Additionally, the effects of heat release and scramjet structure on net thrust have been discussed. The present results and the developed analytical method can provide guidance for the design and optimization of high-performance dual-mode scramjets.
\end{abstract}

Keywords Dual-mode scramjet - Engine performance . Thrust - Optimization · Heat release distribution - Simulated annealing algorithm

Li-Hong Chen

lhchen@imech.ac.cn

1 State Key Laboratory of High-Temperature Gas Dynamics, Institute of Mechanics, Chinese Academy of Sciences, Beijing 100190, China

\section{Introduction}

During the past 50 years, the dual-mode scramjet has become a promising engine for hypersonic propulsion systems [1]. It can operate and maintain desirable performance within a wide range of flight conditions. Theoretical [2] and experimental efforts $[3,4]$ have been made to gain varied heat release and structure schemes for dual-mode combustion. However, an efficient analytical method applicable to the whole process of dual-mode combustion and to the optimization of engine performance is demanding.

Previous design and analytical methods [5-10] were mostly concentrated on a supersonic combustion mode. In the 1960s, the integral analytical approach was initially used to analyze engine cycle aerothermodynamics in scramjets [5]. In this approach, the engine was divided into four components: inlet, isolator, combustor, and nozzle. Each component was analyzed separately but quasi-one-dimensional representation of flow for defined control volumes can be made in a global manner. This integral quasi-one-dimensional approach has been largely developed and can be classified into two categories according to the type of equations. Due to the high efficiency, quasi-one-dimensional aerothermodynamic ordinary differential equations (ODEs) have been widely adopted in the combustor section with different energy release models. These models can be obtained from pre-given experimental data or theoretical assumptions, such as the Crocco power law relationship [6-8]. Alternatively, the energy release is linearly related to the fuel-air mixing rate of normal injections $[9,10]$. However, analytical models based on the ODEs cannot be applied to solve subsonic combustion, as the problem of singularity exists in the original formalism. Although the unsteady quasi-1D 
model based on Euler equations [11] can capture the position of normal shock automatically, normal shock is not favored in the dual-mode system due to the inclination of inlet unstart. However, the oblique shock train, which is more common in the dual-mode scramjet, cannot be predicted by the unsteady quasi-1D model. As such, a reliable analytical model is required to offer consistent solutions for dual-mode combustion.

The analytical method adopted in the present work tends to overcome the shortcomings of previous analytical models by proposing an $\mathrm{X}$-shock model as a strength-adaptable isolator model and combining it with the quasi-1D combustor flow calculation (ODEs) through matching combustion back pressure automatically [12]. It was validated by a series of experimental data [13] and can be applied as a tool to analyze the engine performance in different combustion modes.

The key for maintaining high performance for a dualmode scramjet within a wide flight range is that it can produce high thrust by operating in different modes. It is known that subsonic combustion can offer higher thrust at a flight Mach number of 4-6, as compared to supersonic combustion; while the thrust of the latter can exceed that of the former at a flight Mach number greater than 6 [14]. Hence, an analytical study focusing on the flight Mach number of 6 is of great importance in understanding the dualmode combustion process and also in practical applications [15].

Targeted at the maximum engine net thrust, optimization approaches including the genetic algorithm and the simulated annealing algorithm have been used to achieve optimal heat releasing schemes and engine geometries for dual-mode scramjets [16-18]. However, most previous studies simply choose the design point of inlet for the corresponding flight condition and use the designed inlet parameters as initials of combustor calculations without a thorough analysis. In addition, the trend of heat releasing schemes and engine geometries with the variation of inlet conditions has yet to be studied.

Thus, the present work focuses on the engine performance at the flight Mach number of 6 . Instead of pre-given inlet parameters, the current work includes a simplified 2D inlet model to generate different inlet conditions. Aiming at the maximum net thrust, the simulated annealing algorithm is adopted to optimize the thrust result, which is obtained from the modified quasi-1D analytical model developed by the authors [12]. An optimal combustion mode and inlet condition has been finally chosen for the given flight condition. The trend of heat releasing distributions and engine structures with varied inlet conditions has been discussed. The results can provide guidance for the future design of high-performance dual-mode scramjets.

\section{Model description}

For the dual-mode scramjet studied in the present work, the length in the spanwise direction is kept the same, and thus the engine can be simplified as a 2D model, as shown in Fig. 1. The engine consists of inlet, isolator, combustor, and nozzle. The total length and nozzle height are labeled as $L_{\mathrm{e}}$ and $H_{\mathrm{e}}$, respectively. The inlet height is chosen to be half of the nozzle height for the present scramjet model. All horizontal and vertical structural parameters have been normalized by $L_{\mathrm{e}}$ and $H_{\mathrm{e}}$, respectively.

As illustrated in Fig. 1, the preliminary analysis for the dual-mode scramjet in the present work consists of two parts, which are redrawn in Figs. 2 and 3, respectively. Part I is the calculation of inlet parameters. As shown in Fig. 2, the calculation is based on a four-wave simplified method with the assumption that the first three oblique shocks focus at the leading edge of the cowl and the wall friction is neglected. Given the flight condition of the incoming flow and Mach numbers at the exit of the inlet (i.e., the isolator entrance Mach number $M a_{\text {Iso }}$ ), different inlet structural and aerodynamic parameters can be obtained.

As illustrated in Fig. 3, Part II includes isolator, combustor, and nozzle. The initial flow conditions of Part II are obtained from the outlet conditions of Part I (e.g., $M a_{\text {Iso }}$ ). Targeted at the highest engine net thrust ( $\left.F_{\text {int,hot }}\right)$, an optimization procedure based on the quasi-1D analysis model was conducted.

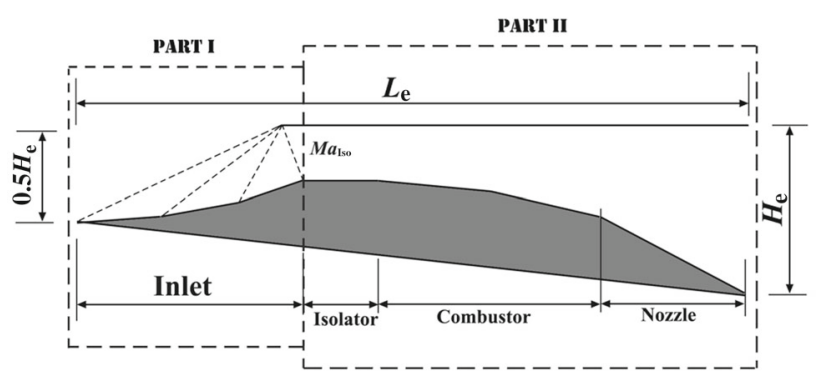

Fig. 1 Scheme of parameterized dual-mode scramjet engines

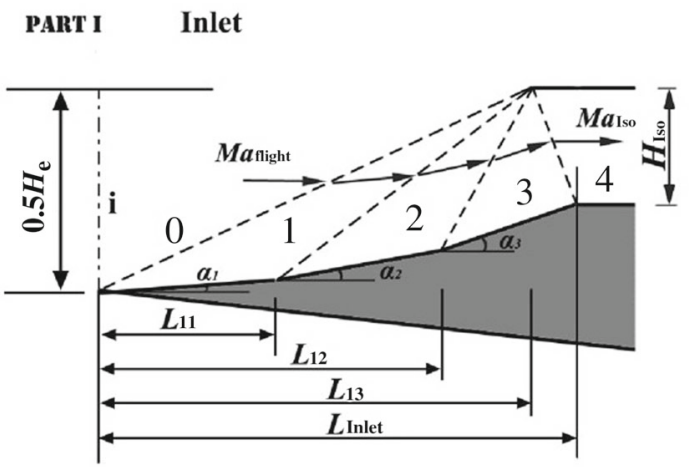

Fig. 2 Scheme of calculation of inlet parameters 


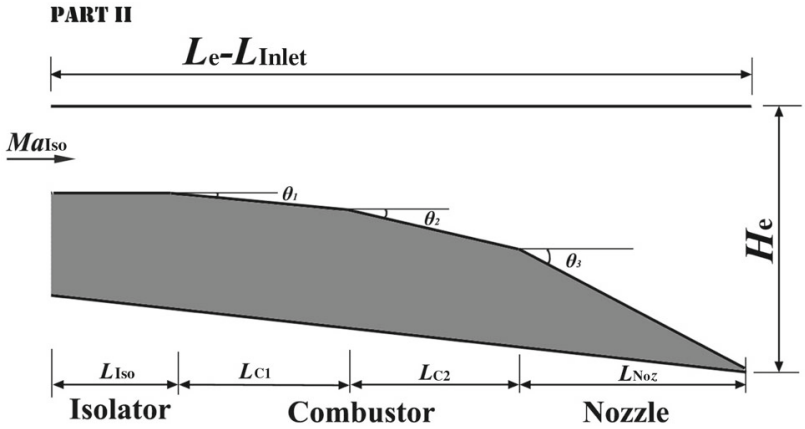

Fig. 3 Scheme of parameterized engine duct

Engine net thrust $F_{\text {int,hot }}$ expressed as Eq. (1) represents the difference of impulse functions between the inlet and outlet of the engine,

$F_{\text {int,hot }}=(\dot{m} v+p A)_{\mathrm{e}}-(\dot{m} v+p A)_{\mathrm{i}}$,

where the subscripts e and i represent parameters at the outlet cross section of nozzle and inlet cross section of inlet, respectively. stands for the flow rate of air flow. The flow rate of fuel is much smaller than that of air and is thus neglected in the calculation of the engine net thrust.

Additionally, specific impulse $\left(I_{\mathrm{S}}\right)$, as an important indicator of engine performance, as shown in Eq. (2), was also calculated,

$I_{\mathrm{S}}=F_{\text {int,hot }} /\left(\dot{m}_{\mathrm{f}} g\right)$,

where $\dot{m}_{\mathrm{f}}$ represents the flow rate of fuel flow.

The optimized structural and heat releasing parameters as well as the optimal combustion mode for each inlet condition $\left(M a_{\text {Iso }}\right)$ can be obtained when the optimization process converges. The quasi-one-dimensional analysis model adopted in the optimization procedure is described briefly in Sect. 2.2.

Figure 4 shows the flowchart of the whole calculation. Specifically, the calculation corresponding to Part I was conducted straightforwardly, while the optimization process for Part II involved interactions of parameters between the quasi-one-dimensional model and the optimization method,

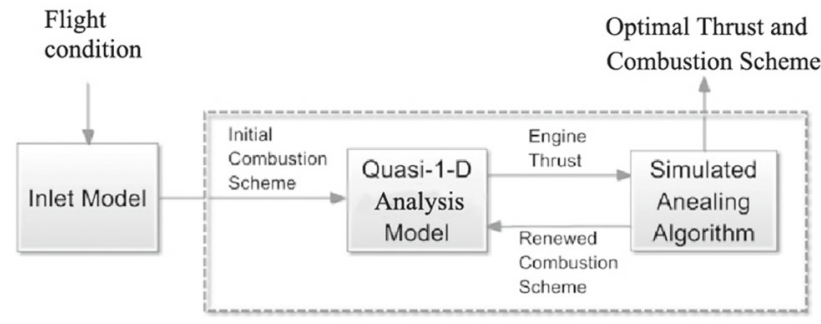

Fig. 4 Flowchart of the analytical process for parameterized dualmode scramjets which is a simulated annealing algorithm in the present work. Details of the methodology are addressed in Sects. 2.1 and 2.2.

\subsection{Inlet model}

Given the conditions of incoming airflow, flow properties at the exit of inlet are calculated by a four-shock-wave strategy. As mentioned earlier, the flight Mach number $\left(M a_{\text {flight }}\right)$ is chosen as 6 in the present work. As shown in Fig. 2, the inlet model contains five sub sections, named as 0 to 4 . The gas is assumed to be a perfect gas. Three other assumptions used in the inlet calculation are as follows,

(1) The distance between fore-body leading edge and cowl lip $L_{\mathrm{I} 3}$ is fixed and the $L_{\mathrm{I} 3} / L_{\mathrm{e}}$ equals 0.320 . As a result, the intensity of the first wave separating section 0 and 1 has been designed beforehand based on engineering experience $\left(\alpha_{1}=4.64\right)$;

(2) The second and third shock waves share the same compression ratio of pressure;

(3) The fourth wave guarantees the designed isolator Mach number $\left(M a_{\text {Iso }}\right)$ and the flow afterwards turns to the original streamwise direction.

In the present work, varied inlet conditions are considered for the flight Mach number of 6 . In order to find the best designed isolator entrance Mach number and evaluate the whole process of dual-mode combustion, the isolator entrance Mach number ranges from 1.5 to 3.5, as listed in Table 1. According to the four-wave method, the structure and flow properties in the inlet can be determined for each $M a_{\text {Iso }}$. Additionally, as the viscosity is considered in the analysis of a downstream combustor, the thickness of boundary layer has been considered to be a tenth of the inlet length for the sake of simplification of the analysis. $H_{\text {Iso }}$ and $H_{\mathrm{I} \text {, vis }}$ represent the isolator entrance height with and without the consideration of flow viscosity, respectively. Hence, the four-wave system provides different initial flow conditions for the quasi-onedimensional optimization and analysis of Part II downstream.

Table 1 Normalized structural parameters of inlet at $M a_{\text {flight }}=6$

\begin{tabular}{llll}
\hline$M a_{\text {Iso }}$ & $L_{\text {Inlet }} / L_{\mathrm{e}}$ & $H_{\text {Iso }} / H_{\mathrm{e}}$ & $H_{\mathrm{I}, \mathrm{vis}} / H_{\mathrm{e}}$ \\
\hline 1.5 & 0.331 & 0.028 & 0.050 \\
2.0 & 0.336 & 0.031 & 0.054 \\
2.5 & 0.345 & 0.040 & 0.063 \\
3.0 & 0.360 & 0.055 & 0.079 \\
3.5 & 0.382 & 0.078 & 0.105 \\
\hline
\end{tabular}




\subsection{Quasi-1D optimization and analysis}

For each inlet condition calculated above, the optimal engine net thrust has been obtained from the optimization process based on the simulated annealing algorithm. Optimized parameters for engine heat releasing distributions and structural configurations are described in Sect. 2.2.1. The quasi-1D analytical model adopted to calculate engine net thrust is briefly introduced in Sect. 2.2.2.

\subsubsection{Parameters for optimization}

As shown in Fig. 3, the isolator is a straight section and the

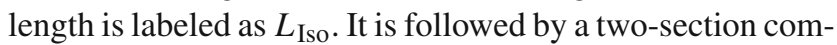
bustor and nozzle with different divergence angles. The total length, the height of the isolator entrance, and the height of nozzle exit are fixed. The heat release, occurring in the twosection combustor, is assumed to be uniform for each section and has the same length as the combustor section. The aim of the optimization is to provide the heat releasing as well as structural parameters that could generate the highest engine net thrust. Hence, nine parameters are evaluated during the optimization procedure. They are the length of the isolator $\left(L_{\text {Iso }}\right)$, the length and divergence angle of combustor section one $\left(L_{\mathrm{C} 1}\right.$ and $\left.\theta_{1}\right)$, the length and divergence angle of combustor section two $\left(L_{\mathrm{C} 2}\right.$ and $\left.\theta_{2}\right)$, the length and divergence angle of the nozzle ( $L_{\mathrm{Noz}}$ and $\left.\theta_{3}\right)$ and the equivalent combustion efficiencies for two combustion sections ( $\eta_{\text {eq1 }}$, and $\eta_{\text {eq2 } 2}$ ). During the process of optimization, several restrictions were applied to guarantee rational solutions, as written in Eq. 3. The seven optimal normalized structural parameters for the combustor and nozzle in the current study are listed in Table 2.

The simulated annealing algorithm [19] was adopted as the optimization method in this work. It is a Monte-Carlo method, simulating the physical process of annealing. The most global optimal result with modified probability of acceptance was adopted in this process.

$L_{\text {Iso }}+L_{\mathrm{C} 1}+L_{\mathrm{C} 2}+L_{\mathrm{Noz}}=L_{\mathrm{e}}-L_{\text {inlet }}$,

$L_{\mathrm{C} 1} \times \tan \theta_{1}+L_{C 2} \times \tan \theta_{2}+L_{N o z} \times \tan \theta 3=H_{\mathrm{e}}-H_{\mathrm{I}, \mathrm{vis}}$,

$0 \leq \theta_{1} \leq \theta_{2} \leq \theta_{3}$,

Table 2 Normalized structural parameters of inlet at $M a_{\text {flight }}=6$

\begin{tabular}{lcclllll}
\hline$M a_{\text {Iso }}$ & $\frac{L_{\mathrm{ISo}}}{L_{\mathrm{e}}}$ & $\frac{L_{\mathrm{C} 1}}{L_{\mathrm{e}}}$ & $\theta_{1}$ & $\frac{L_{\mathrm{C} 2}}{L_{\mathrm{e}}}$ & $\theta_{2}$ & $\frac{L_{\mathrm{Noz}}}{L_{\mathrm{e}}}$ & $\theta_{3}$ \\
\hline 1.5 & 0.187 & 0.127 & 1.238 & 0.041 & 2.842 & 0.315 & 23.035 \\
2.0 & 0.175 & 0.116 & 0.922 & 0.052 & 1.338 & 0.321 & 22.836 \\
2.5 & 0.136 & 0.132 & 0.504 & 0.088 & 0.560 & 0.299 & 24.255 \\
3.0 & 0.255 & 0.065 & 0.822 & 0.029 & 0.927 & 0.291 & 24.559 \\
3.5 & 0.239 & 0.053 & 6.100 & 0.051 & 7.049 & 0.275 & 23.378 \\
\hline
\end{tabular}

$\eta_{\text {eq } 1}+\eta_{\text {eq2 }} \leq 1$

$0 \leq \eta_{\text {eq } 1} \leq 1$,

$0 \leq \eta_{\mathrm{eq} 2} \leq 1$.

\subsubsection{Quasi-1D analysis model}

A modified quasi-1D analytical method proposed by the authors [12] has been adopted in this work. First, it is based on aerodynamic quasi-one-dimensional conservation equations of mass, momentum, and energy as well as the equation of state andthe Mach number definition equation. These are a series of original ODEs. However, the main characteristic of the modified method is that it includes the $\mathrm{X}$-shock model to simulate the behavior of the shock system in the isolator to facilitate the calculation of the whole process of a dual-mode scramjet. In addition, restrictions on heat release have been used to avoid overestimating heat release in the optimization process.

\section{(1) X-shock model}

As shown in Fig. 5, the flow calculation consists of three parts: the upstream is a 2D shock structure (X-shock) in order to balance the shock and combustion interaction, the downstream is a quasi-1D continuous flow with heat releasing model to simulate combustion. Conservations of mass, momentum, and energy are applied for the transition from the $2 \mathrm{D} \mathrm{X}$-shock model to the $1 \mathrm{D}$ heat releasing flow. The $\mathrm{X}$-shock model consists of two pairs of oblique shocks with symmetry in the vertical direction that simulates the precombustion shock train structure originated in the isolator. The parameters in the $\mathrm{X}$-shock region are resolved by equations of conservation laws and the shock strength is determined due to the back pressure of combustion downstream. The length of the precombustion shock train is obtained through

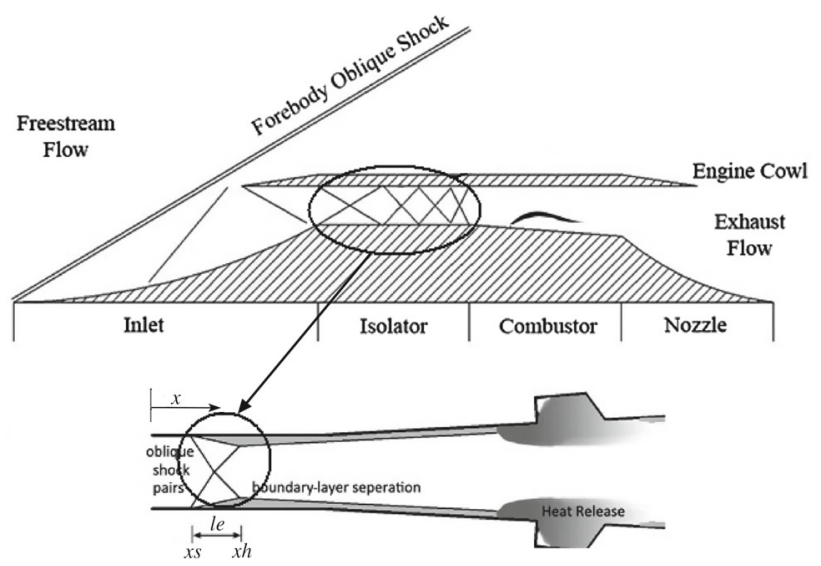

Fig. 5 Sketch of the dual-mode scramjet engine and "X-shock" model [12] 
experimental semi-empirical equations as described in Billig's milestone work [20]. The interactive process between $\mathrm{X}$-shock and quasi-1D models guarantees the ability of the present method to calculate the whole dual-mode combustion process, ranging from supersonic combustion mode, to dual-mode, and then to subsonic combustion mode.

\section{(2) Heat release restriction model}

In order to unify the scaling of heat release, the conception of equivalent combustion efficiency $\left(\eta_{\mathrm{eq}}\right)$ was adopted [5] as an optimization parameter instead of a combustion efficiency $\left(\eta_{\mathrm{c}}\right)$. As shown in Eq. 4, equivalent combustion efficiency $\left(\eta_{\text {eq }}\right)$ equals the product of combustion efficiency $\left(\eta_{\mathrm{c}}\right)$ and the equivalence ratio $(\phi)$, representing the combustion efficiency at the stoichiometric fuel/air ratio.

$\eta_{\mathrm{eq}}=\eta_{\mathrm{c}} \times \phi$.

To expedite the calculation, a one-step global reaction has been employed as the combustion model and written as,

$\eta_{\text {eq }}($ fuel $)+$ air $\rightarrow$ products $+Q$,

where $Q$ stands for the heat release generated by chemical reactions.

Additionally, when the temperature is comparatively high, the dissociation reaction of oxygen, nitrogen, and reaction products can occur, resulting in a reduction of the whole heat release. The effect of gas dissociation is included in the present work by defining a new expression of heat release, denoted as $Q^{\prime}$. As shown in Eq. (6), $Q^{\prime}$ relies on equivalent combustion efficiency $\left(\eta_{\mathrm{eq}}\right)$ and static temperature $(T)$ and also static pressure $(p)$. Since the static pressure of combustion studied in our work is in the range of $1 \mathrm{~atm}$ to $10 \mathrm{~atm}$ $\left(1 \mathrm{~atm}=1.01 \times 10^{5} \mathrm{~Pa}\right)$ and the dependency on static pressure $(p)$ is modest, $Q^{\prime}$ is linearly interpolated in the pressure range, as written in Eq. (6). In particular, two sub functions $f_{p 1}\left(\eta_{\mathrm{eq}}, T\right)$ and $f_{p 10}\left(\eta_{\mathrm{eq}}, T\right)$ corresponding to the upper and lower limits of static pressure were fitted according to the data calculated by CHEMKIN with dissociation reactions included. For kerosene $\left(\mathrm{C}_{12} \mathrm{H}_{24}\right)$ studied in this work, $f_{p 1}\left(\eta_{\mathrm{eq}}, T\right)$ and $f_{p 10}\left(\eta_{\mathrm{eq}}, T\right)$ are shown as Eqs. (7) and (8). This ad hoc model affects static temperature significantly in intense combustion.

$$
\begin{aligned}
& Q^{\prime}=f\left(\eta_{\mathrm{eq}}, T, p\right) \\
& \quad=\frac{p}{9} f_{p 10}\left(\eta_{\mathrm{eq}}, T\right)+\frac{(10-p)}{9} f_{p 1}\left(\eta_{\mathrm{eq}}, T\right), \\
& f_{p 1}\left(\eta_{\mathrm{eq}}, T\right)=\left(7.966-0.02192 T-3.553 \eta_{\mathrm{eq}}\right. \\
& \quad+2.636 \times 10^{-5} T^{2}+0.01091 T \eta_{\mathrm{eq}}+0.9845 \eta_{\mathrm{eq}}^{2}
\end{aligned}
$$

$$
\begin{aligned}
&-1.482 \times 10^{-8} T^{3}-1.105 \times 10^{-5} T^{2} \eta_{\mathrm{eq}}-1.194 \times 10^{-4} T \eta_{\mathrm{eq}}^{2} \\
&-3.037 \eta_{\mathrm{eq}}^{3}+3.909 \times 10^{-12} T^{4}+4.494 \times 10^{-9} T^{3} \eta_{\mathrm{eq}} \\
&-8.943 \times 10^{-7} T^{2} \eta_{\mathrm{eq}}^{2}+3.534 \times 10^{-3} T \eta_{\mathrm{eq}}^{3}-3.839 \times 10^{-16} T^{5} \\
&-5.964 \times 10^{-13} T^{4} \eta_{\mathrm{eq}}+2.598 \times 10^{-10} T^{3} \eta_{\mathrm{eq}}^{2} \\
&-\left.8.45 \times 10^{-7} T^{2} \eta_{\mathrm{eq}}^{3}\right) \quad(\mathrm{kJ} / \mathrm{kg} \cdot \mathrm{K}), \\
& f_{p 1}\left(\eta_{\mathrm{eq}}, T\right)=\left(5.028-0.01101 T-7.408 \eta_{\mathrm{eq}}\right. \\
& \quad+1.183 \times 10^{-5} T^{2}+0.01564 T \eta_{\mathrm{eq}}+5.265 \eta_{\mathrm{eq}}^{2} \\
&--5.976 \times 10^{-9} T^{3}-1.152 \times 10^{-5} T^{2} \eta_{\mathrm{eq}}-0.007284 T \eta_{\mathrm{eq}}^{2} \\
&-2.452 \eta_{\mathrm{eq}}^{3}+1.434 \times 10^{-12} T^{4}+3.581 \times 10^{-9} T^{3} \eta_{\mathrm{eq}} \\
&+2.88 \times 10^{-6} T^{2} \eta_{\mathrm{eq}}^{2}+0.002718 T \eta_{\mathrm{eq}}^{3}-1.29 \times 10^{-16} T^{5} \\
&-3.815 \times 10^{-13} T^{4} \eta_{\mathrm{eq}}-3.608 \times 10^{-10} T^{3} \eta_{\mathrm{eq}}^{2} \\
&\left.-6.067 \times 10^{-7} T^{2} \eta_{\mathrm{eq}}^{3}\right) \quad(\mathrm{kJ} / \mathrm{kg} \cdot \mathrm{K}) .
\end{aligned}
$$

It is also worth mentioning that the ODEs were solved by the ODE15s function of MATLAB based on the Gear algorithm to avoid the stiffness. In addition, some posterior conditions and mathematical treatments have been set into the model in order to guarantee realizability. For instance, the calculated length of the shock train should be shorter than that of the isolator to avoid the engine unstart. Other details of the multi-mode quasi-one-dimensional method are available in Ref. [21] and Sect. 2 of Ref. [12].

\section{Results and discussion}

As mentioned in Sect. 2, the methodology can offer optimal parameters for the parameterized engine under each inlet condition. By comparing the results for different inlet conditions, the best inlet condition and optimal parameters can be chosen for the flight Mach number of 6 .

The key for the maximum thrust includes two points: high efficiency of combustion itself and maximum transition to thrust. The former is related to the heat releasing scheme and the latter is to refer to the engine geometries. The combustor is the core part, which ensures efficient combustion. The heat release is highly coupled with the engine configuration, both affecting the maximum net thrust.

The total length and height of the engine are fixed. The length and divergence angle of each section are obtained from the optimizations. The isolator is the component of drag, which is used to balance the interaction between shock wave and combustion. It is noted that the length of isolator should be long enough to avoid the engine unstart.

\subsection{Optimal parameters for varied inlet conditions}

The results for five different isolator entrance Mach numbers $\left(M a_{\text {Iso }}\right)$ were analyzed in order to understand the optimal heat 
release distributions and the corresponding geometries under each inlet condition.

The distributions of static pressure and Mach number along the engine axial direction for the optimal combustion are shown in Fig. 6a, b, respectively. As shown in Fig. 6a, the static pressure along the engine duct is normalized by the corresponding isolator entrance static pressure $\left(p_{\mathrm{I}}\right)$. Inspection of the figures reveals that the $M a_{\text {Iso }}$ of 2.5 is the critical point. When $M a_{\text {Iso }}$ is lower than 2.5, the pressure rising position propagates upstream with the increasing $M a_{\text {Iso }}$. The pressure distributions have similar shapes but become plumper with the increasing $M a_{\mathrm{Iso}}$. Correspondingly, the subsonic combustion tends to occur at a constant-Mach number near 0.6, as shown in Fig. 6b. If $M a_{\text {Iso }}$ increases further, the isolator cannot afford to have the similar combustion mode occur. That is, the isolator is not long enough to hold intense combustion. Hence, the largest propagation distance of the shock occurs at the $M a_{\text {Iso }}$ of 2.5. Then the shock train retreats with the increasing $M a_{\text {Iso }}$. When $M a_{\text {Iso }}$ equals 3.0, the distributions of pressure and Mach number are no longer uniform in the
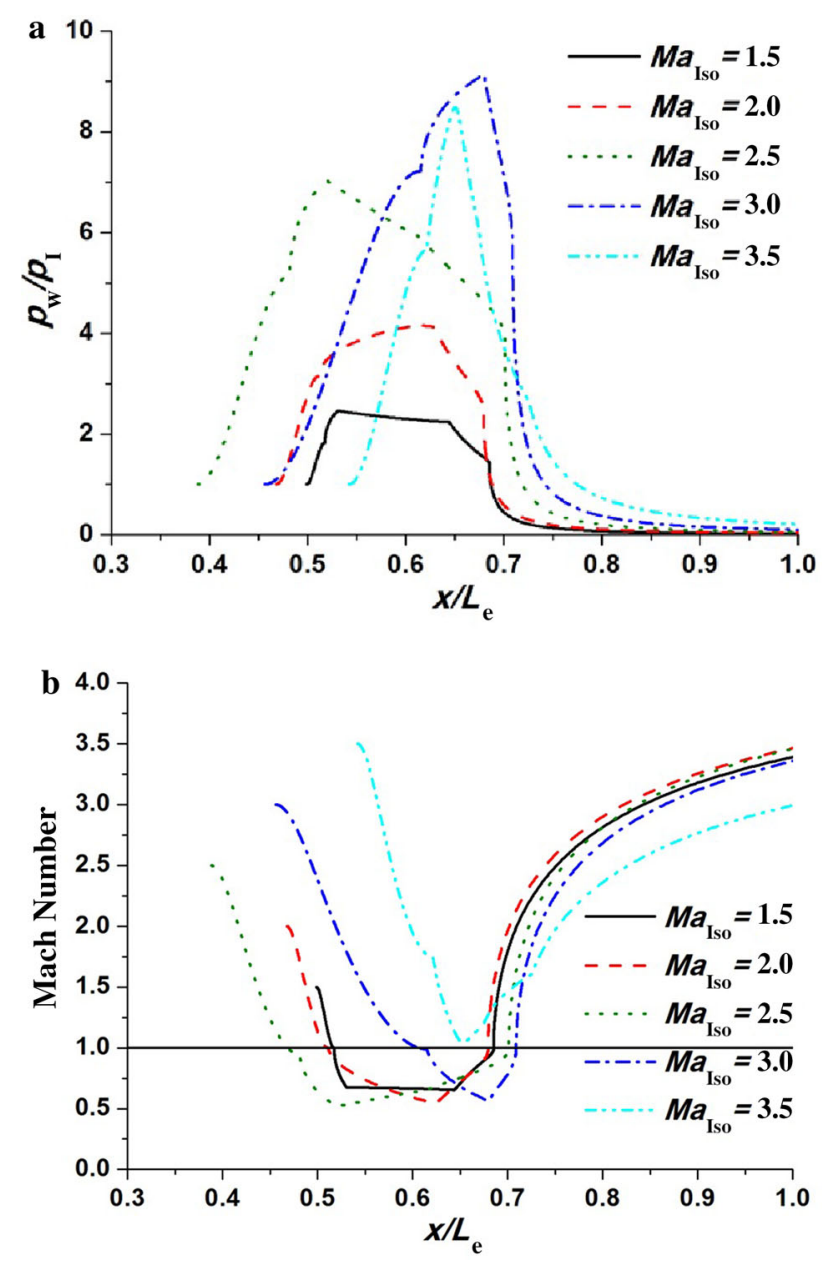

Fig. 6 Optimal distributions for different $M a_{\text {Iso }}$ a Optimal pressure distributions. b Optimal Mach number distributions combustor section. The pressure distribution is rather sharp although the subsonic combustion mode is still maintained. When $M a_{\text {Iso }}$ increases to 3.5, the length of the isolator cannot support the propagation distance of the shock train for subsonic combustion. Thus, there is no subsonic combustion solution for this case, the optimal combustion mode is supersonic combustion and the lowest Mach number in the combustor section is near one.

In addition to the static pressure and Mach number distributions, the optimal heat release distributions and engine geometries are also of great importance. As shown in Fig. 7, equivalent combustion efficiency $\left(\eta_{\mathrm{eq} 1}\right.$ or $\left.\eta_{\mathrm{eq} 2}\right)$ has been normalized by the corresponding length of the combustor section $\left(L_{\mathrm{C} 1}\right.$ or $\left.L_{\mathrm{C} 2}\right)$. The ratio of $\eta_{\mathrm{eq}} / L_{\mathrm{c}}$ can demonstrate the intensity of combustion along the combustor. As shown in Fig. 7, different combustion modes have diverse heat releasing schemes. When $M a_{\text {Iso }}$ is relatively low, ranging from 1.5 to 2.0 , only an intense heat release in the second section can form the thermal throat at the end of the combustor. The subsonic combustion mode could provide efficient combustion and the maximum thrust. This conclusion is also coincidence with the concept of ramjet design. When $M a_{\text {Iso }}$ goes up to 2.5 , the heat release distribution is rather smooth, and the length of heat release is much longer. When $M a_{\text {Iso }}$ increases to 3.0, the incoming flow speed is so high that only a very sharp heat release could form subsonic combustion. For the case with $M a_{\text {Iso }}$ of 3.5 , the heat release is rather stronger in the first combustion section and, consequently, supersonic combustion occurs. Comparing the five optimal heat release distributions, the $M a_{\text {Iso }} 2.5$ case has the most feasible heat releasing scheme with relatively low intensity $\left(\eta_{\mathrm{eq}} / L_{\mathrm{c}}\right)$.

Additionally, the optimal results of engine configurations for the five inlet Mach numbers are shown in Fig. 8. Since the combustor section is relatively shorter in our results, differences among the five cases are modest in a view of the whole

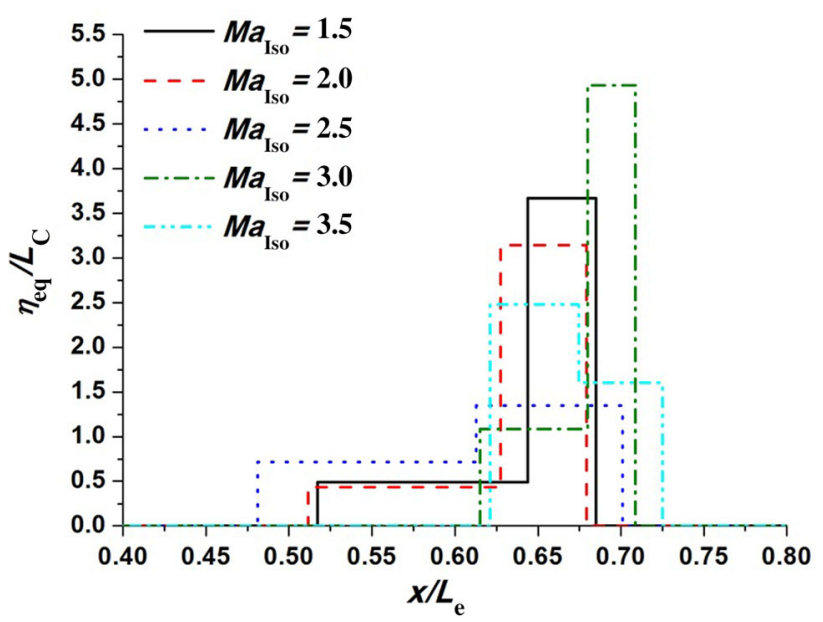

Fig. 7 Equivalent heat release distributions for different $M a_{\text {Iso }}$ 


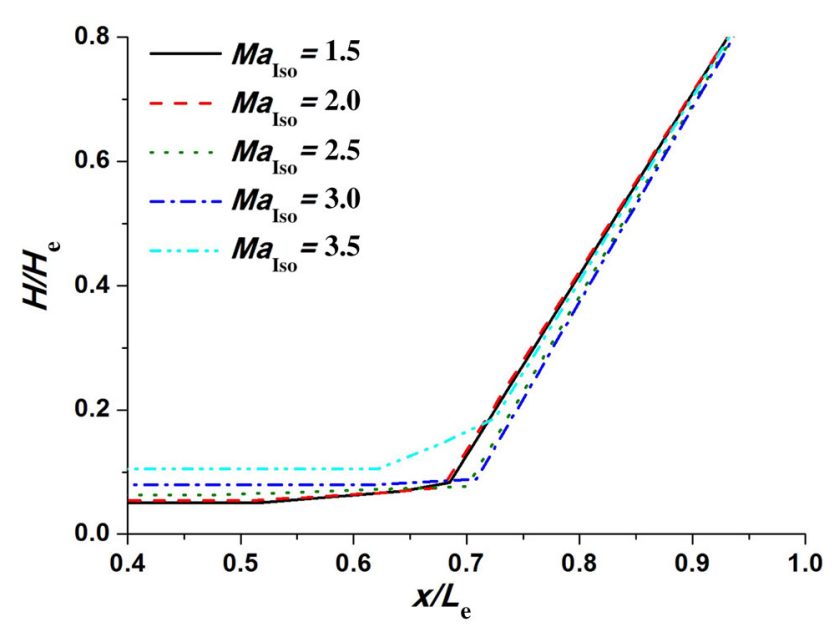

Fig. 8 Normalized engine structures for different $M a_{\text {Iso }}$

Table 3 Optimal combusting parameters for different isolator conditions at a flight Mach number of 6

\begin{tabular}{lllll}
\hline$M a_{\text {Iso }}$ & $\eta_{\text {eq1 }}$ & $\eta_{\text {eq2 }}$ & $I_{S}\left(\mathrm{~s}^{-1}\right)$ & Mode \\
\hline 1.5 & 0.286 & 0.694 & 1140 & Subsonic \\
2.0 & 0.232 & 0.748 & 1180 & Subsonic \\
2.5 & 0.435 & 0.545 & 1193 & Subsonic \\
3.0 & 0.323 & 0.657 & 1171 & Subsonic \\
3.5 & 0.608 & 0.372 & 1123 & Supersonic \\
\hline
\end{tabular}

engine structure. However, different divergence angles of the combustor walls can be readily observed. In particular, the divergence angle of the combustor in the supersonic combustion mode is much larger than that in the subsonic combustion mode in order to accommodate more energy release. Furthermore, the divergence angle slightly decreases with the increase of $M a_{\text {Iso }}$. It can be explained by different heat releasing characteristics between supersonic and subsonic flows. The flow speed increases with the divergence angle, resulting in a high thrust in the supersonic combustor; while a subsonic combustor with a smaller divergence angle could guarantee more intense heat release and the formation of the thermal throat.

\subsection{Optimal net thrust}

Table 3 lists the optimal results for the five inlet conditions, including the equivalence of combustion efficiency for the two heat-releasing regions, the specific impulse, and the corresponding combustion mode.

In order to choose a desirable combustion mode and isolator entrance condition, five optimal net thrust values are compared in Fig. 9. In addition to the assumption of viscous flow with modified heat release (see Sect. 2.2.2) adopted in the above calculation, two more simplified cases (inviscid

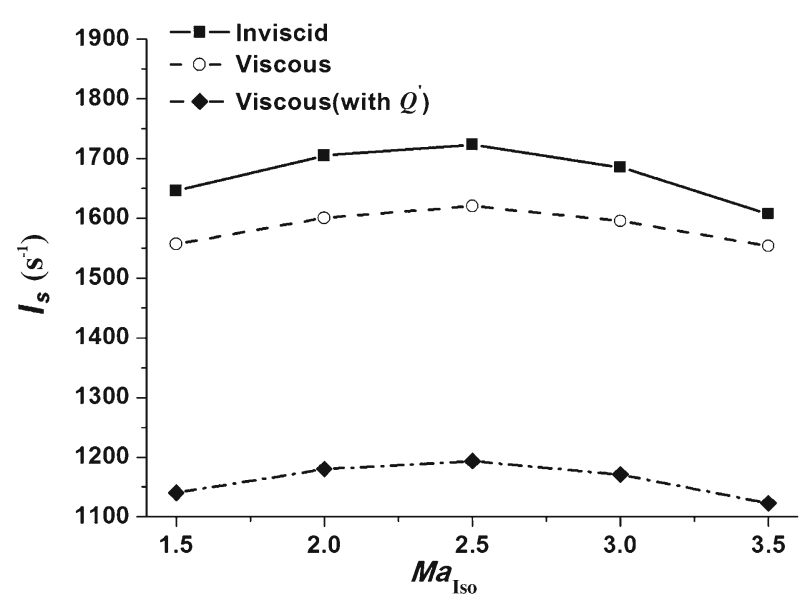

Fig. 9 Comparison of specific impulse for different $M a_{\text {Iso }}$

flow, viscous flow without modification on heat release) are included for comparison. As shown in Fig. 9, the overall tendency is the same despite different assumptions. The highest net thrust occurs when $M a_{\text {Iso }}$ equals 2.5 . The viscous boundary layer and heat release restriction model, which simulates dissociation effects, both cause extra heat loss in combusting flows. Hence, the case considering both of these effects offers the lowest net thrust distribution. On the other hand, in order to estimate more accurate engine performance, these two effects cannot be neglected in the optimization process.

To sum up, the parameterized dual-mode scramjet at the flight Mach number of 6 is suitable to operate at subsonic combustion when the isolator entrance Mach number ranges from 1.5 to 3.0; while supersonic combustion should be chosen when the isolator entrance Mach number equals to 3.5. Comparing the optimal net thrusts, adopting the isolator Mach number of 2.5 is the most desirable for the current flight condition.

\section{Conclusions}

An optimization process of a dual-mode scramjet at the flight Mach number of 6 was carried out by applying the simulation annealing algorithm and the modified 1D analytical model. The modified quasi-1D model includes an X-shock model to simulate the effect of the shock train and the iterative mechanism between the shock system and combustion guarantees the calculation of different combustion modes in a dual-mode scramjet. Different inlet conditions were obtained based on the four-wave assumption. During the optimization process, the engine net thrust was chosen as the target function, parameters of heat release distributions, and engine structures were investigated.

The results show that the current analytical method can obtain optimal results for different isolator entrance Mach 
numbers. The trend of heat release and combustor structures for the maximum net thrust can be determined. When the aircraft is operated at a flight Mach number of 6, where the performance of supersonic and subsonic combustion compete, the optimal combustion mode changes from a nearly constant-Mach subsonic combustion mode to supersonic combustion mode with the increase of the isolator entrance Mach number $\left(M a_{\text {Iso }}\right)$. Based on the present results, the engine can achieve the highest net thrust at the isolator entrance Mach number of 2.5. It is also noted that the heat release scheme for $M a_{\text {Iso }} 2.5$ case is comparatively realistic for practical applications.

The analytical method used in the present work can offer a consistent analysis of the performance of dual-mode combustion. The present results provide useful guidance for the design and optimization of dual-mode scramjets.

Acknowledgments This work was supported by the National Natural Science Foundation of China (Grant 11002148).

\section{References}

1. Curran, E.T.: Scramjet engines: the first 40 years. J. Propuls. Power 17, 1138-1148 (2001)

2. Heiser, W.H., Pratt, D.T., Deley, D.H.: Hypersonic Airbreathing Propulsion. American Institute of Aeronautics and Astronautics Inc, Washington, D.C. (1994)

3. Dessornes, O., Scherrer, D.: Tests of the JAPHAR dual mode ramjet engine. Aerosp. Sci. Technol. 9, 211-221 (2005)

4. Canell, K., Hass, N., Storch, A., Gruber, M.: HIFiRE direct-connect rig (HDCR) phase I scramjet test results from the NASA langley arc-heated scramjet test facility. In: 17th AIAA International Space Planes and Hypersonic Systems and Technologies Conference, AIAA-2011-2248 (2011)

5. Waltrup, P.J., Billig, F.S., Stockbridge, R.D.: A procedure for optimizing the design of scramjet engines. J. Spacecr. Rockets 16, 163-172 (1979)

6. Pinckney, S.Z.: Integral Performance Predictions for Langley Scramjet Engine Module. NASA TM-X-74038 (1978)

7. Henry, J.R., Anderson, G.Y.: Design considerations for the airframe integrated scramjet. In: 1st International Symposium on Air Breathing Engine, Marseille, June 1972; also NASA TMX-2895 (1973)
8. Billig, F.S., Dugger, G.L.: The interaction of shock waves and heat addition in the design of supersonic combustors. In: Twelfth Symposium (International) on Combustion, The Combustion Institute, Pittsburgh, pp. 1125-1134 (1969)

9. O'Brien, T., Starkey, R., Lewis, M.: Quasi-one-dimensional highspeed engine model with finite-rate chemistry. J. Propuls. Power 17, 1366-1374 (2001)

10. Ferguson, F., Dhanasar, M.: A model for the design and analysis of thrust optimized ccramjets. In: 16th AIAA/DLR/DGLR International Space Planes and Hypersonic Systems and Technologies Conference, AIAA 2009-7337 (2009)

11. Bussing, T.R.A., Murman, E.M.: A One-Dimensional Unsteady Model of Dual Mode Scramjet Operation. AIAA Paper 83-0422 (1983)

12. Tian, L., Chen, L.H., Chen, Q., et al.: Quasi-one-dimensional multimodes analysis for dual-mode scramjet. J. Propuls. Power 30, 1559-1567 (2014)

13. Li, F., Yu, X.L., Gu, H.G., et al.: Measurement of flow parameters in a scramjet combustor based on near-infrared absorption. Chin. J. Theor. Appl. Mech. 43, 1061-1067 (2011). (in Chinese)

14. Daines, R., Segal, C.: Combined rocket and airbreathing propulsion systems for space-launch applications. J. Propuls. Power 14, 605612 (1998)

15. Castro, J., Brink, C., Mutzman, R., et al.: X51 session. In: Proceedings of 46th American Institute of Aeronautics and Astronautics Joint Propulsions Conf, American Institute of Aeronautics and Astronautics, Reston (2010)

16. Cao, R.F., Chang, J.T., Bao, W., et al.: Analysis of combustion mode and operating route for hydrogen fueled scramjet engine. Int. J. Hydrog. Energy 38, 5928-5935 (2013)

17. Chen, Q., Chen, L.H., Gu, H.B., et al.: Investigation of the effect and optimization of heat release distributions in the combustor on scramjet performance. J. Propuls. Technol. 30, 135-138 (2009). (in Chinese)

18. Starkey, R.P., Lewis, M.J.: Sensitivity of hydrocarbon combustion modeling for hypersonic missile design. J. Propuls. Power 19, 8997 (2003)

19. Kirkpatrick, G., Gelatt Jr, C.D., Vecchi, M.P.: Optimization by simulated annealing. Science 220, 671-680 (1983)

20. Billig, F.S.: Research on Supersonic Combustion, 10th Aerospace Sciences Meeting and Exhibit, Reno, AIAA-92-0001 (1992)

21. Tian, L.: Investigation on Simplified Modeling and Its Application of Dual-Mode Scramjet Combustor [Master Thesis], University of Chinese Academy of Sciences, Beijing (2013) 